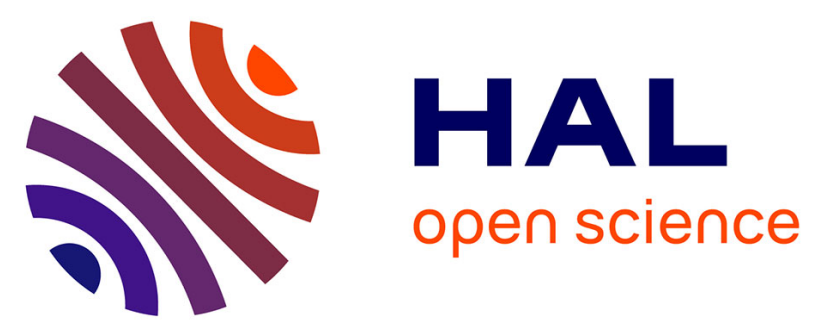

\title{
COMPÉTENCES PSYCHOSOCIALES ET ÉDUCATION THÉRAPEUTIQUE DU PATIENT DIABÉTIQUE DE TYPE 1: UNE REVUE DE LITTÉRATURE
}

David Fonte, Thémis Apostolidis, Marie Claude Lagouanelle-Simeoni

\section{To cite this version:}

David Fonte, Thémis Apostolidis, Marie Claude Lagouanelle-Simeoni. COMPÉTENCES PSYCHOSOCIALES ET ÉDUCATION THÉRAPEUTIQUE DU PATIENT DIABÉTIQUE DE TYPE 1: UNE REVUE DE LITTÉRATURE. Santé Publique, 2014, 26 (763-777). hal-01243026

\section{HAL Id: hal-01243026 \\ https://hal-amu.archives-ouvertes.fr/hal-01243026}

Submitted on 14 Dec 2015

HAL is a multi-disciplinary open access archive for the deposit and dissemination of scientific research documents, whether they are published or not. The documents may come from teaching and research institutions in France or abroad, or from public or private research centers.
L'archive ouverte pluridisciplinaire HAL, est destinée au dépôt et à la diffusion de documents scientifiques de niveau recherche, publiés ou non, émanant des établissements d'enseignement et de recherche français ou étrangers, des laboratoires publics ou privés. 


\section{COMPÉTENCES PSYCHOSOCIALES ET ÉDUCATION THÉRAPEUTIQUE DU PATIENT DIABÉTIQUE DE TYPE 1 : UNE REVUE DE LITTÉRATURE \\ David Fonte, Thémis Apostolidis, Marie-Claude Lagouanelle-Simeoni}

S.F.S.P. $\mid$ « Santé Publique »

2014/6 Vol. 26 | pages 763 à 777

ISSN 0995-3914

Article disponible en ligne à l'adresse :

http://www.cairn.info/revue-sante-publique-2014-6-page-763.htm

\section{!Pour citer cet article :}

David Fonte et al., «Compétences psychosociales et éducation thérapeutique du patient diabétique de type 1 : une revue de littérature », Santé Publique 2014/6 (Vol. 26), p. 763-777.

Distribution électronique Cairn.info pour S.F.S.P..

(C) S.F.S.P.. Tous droits réservés pour tous pays.

La reproduction ou représentation de cet article, notamment par photocopie, n'est autorisée que dans les limites des conditions générales d'utilisation du site ou, le cas échéant, des conditions générales de la licence souscrite par votre établissement. Toute autre reproduction ou représentation, en tout ou partie, sous quelque forme et de quelque manière que ce soit, est interdite sauf accord préalable et écrit de l'éditeur, en dehors des cas prévus par la législation en vigueur en France. Il est précisé que son stockage dans une base de données est également interdit. 


\title{
Compétences psychosociales et éducation thérapeutique du patient diabétique de type 1 : une revue de littérature Psychosocial skills and therapeutic education of patients with type 1 diabetes: a systematic review
}

\author{
David Fonte ${ }^{1}$, Thémis Apostolidis ${ }^{1}$, Marie-Claude Lagouanelle-Simeoni ${ }^{1,2}$
}

\section{$\sqsubset \rightarrow$ Résumé}

Introduction : En éducation thérapeutique, il est établi que les compétences psychosociales doivent être renforcées chez les patients pour les aider à mieux gérer leur maladie et les traitements associés. Actuellement, ce travail est difficilement réalisable en raison de questions conceptuelles, méthodologiques et opérationnelles qui restent en suspens. En particulier, se posent celles de l'identification et de l'évaluation des compétences psychosociales pertinentes à développer.

Objectifs : Il s'agissait de réaliser un inventaire des compétences psychosociales ciblées par les interventions d'éducation et d'identifier les critères utilisés pour objectiver l'acquisition de ces compétences.

Méthode : Une revue de la littérature a été réalisée à partir de 60 articles portant sur l'évaluation d'une intervention d'éducation de patients diabétiques.

Résultats : Les compétences ont été identifiées dans le quart des articles. Elles renvoyaient à la communication et aux relations interpersonnelles, à la prise de décision et à l'esprit critique, et à la gestion de soi. Les articles utilisent davantage de critères d'évaluation de nature médicale que de nature psychosociale.

Discussion: Les compétences psychosociales sont peu explicitées et peu évaluées. Souvent pensées à travers une approche biomédicale, les interventions se focalisent davantage sur les compétences d'autosoin. L'article conclut sur l'intérêt de développer une approche psychosociale pour une meilleure conceptualisation de la notion de compétence sociale.

Mots-clés: Adaptation psychologique; Diabète de type 1; Éducation du patient comme sujet; Psychologie; Revue de la littérature.

\section{$[\rightarrow$ Summary}

Introduction: It has been established that the psychosocial skills of patients need to be strengthened in the context of therapeutic patient education, to help them to more effectively manage their disease and the associated treatments. This intervention is barely feasible at the present time because of unresolved conceptual, methodological and operational problems, particularly problems concerning the identification and evaluation of the psychosocial skills to be developed.

Objectives: This study established an inventory of psychosocial skills targeted by educational intervention, and identified the criteria used to demonstrate acquisition of these skills.

Method: A systematic review of the literature was performed on 60 articles dealing with evaluation of educational intervention in patients with diabetes.

Results: Skills were identified in one quarter of these articles. They referred to communication and interpersonal relations, decisionmaking and critical thinking, and also to coping and selfmanagement. These articles used more often used medical endpoints than psychosocial endpoints.

Discussion: Psychosocial skills are poorly explained and poorly evaluated. Interventions, often based on a biomedical approach, focus more on self-care skills. The paper concludes on the importance of developing a psychosocial approach to provide a better conceptualization of the notion of social skills.

Keywords: Adaptation, psychological; Diabetes mellitus, type 1; Patient education; Psychology; Review.

\footnotetext{
${ }^{1}$ Aix Marseille Université, LPS EA 849 - 13621 Aix-en-Provence - France.

${ }^{2}$ APHM, Conception, Service d'évaluation médicale -13005 Marseille - France. 


\section{Introduction}

Depuis plusieurs années, les compétences psychosociales sont au cœur des politiques de santé publique, en particulier dans le cadre de la prévention. Les compétences psychosociales ont été définies par le psychologue Michael Argyle, sous le terme de social skills, comme des patterns de comportement social qui amènent les individus à devenir socialement compétents, c'est-à-dire capables d'atteindre les buts poursuivis en produisant les effets désirés sur soimême et sur d'autres individus [1]. Pour l'Organisation Mondiale de la Santé (OMS), les compétences psychosociales sont définies comme la capacité de répondre efficacement aux exigences et aux difficultés de l'existence ; elles jouent un rôle important dans la promotion de la santé et du bien-être, notamment lorsque des problèmes de santé sont liés aux capacités des individus à adopter des comportements permettant de répondre efficacement au stress et aux pressions de la vie quotidienne [2].

Appliquées à la pratique de l'éducation thérapeutique, les compétences psychosociales doivent permettre aux patients de s'adapter aux épreuves que suscite la maladie chronique [3]. Ces dernières ont été distinguées par l'OMS en trois catégories : la communication et les relations interpersonnelles (négociation, plaidoyer, empathie, coopération, travail en équipe) ; les prises de décisions et l'esprit critique (esprit analytique et critique, prise de décision, résolution de problème) ; la gestion de soi (confiance en soi, prise de responsabilité, gestion des sentiments et du stress).

Dans le contexte français, le guide méthodologique HAS-INPES pour la structuration d'un programme d'éducation thérapeutique du patient (ETP) pose que les compétences psychosociales doivent faire partie des compétences à développer chez les patients : également appelées compétences d'adaptation, elles ont pour fonction de soutenir l'acquisition des compétences d'autosoins, et reposent sur le développement de l'autodétermination et de la capacité d'agir du patient [4]. Ce guide définit sept compétences d'adaptation : se connaître soi-même; avoir confiance en soi ; savoir gérer ses émotions et maîtriser son stress ; développer un raisonnement créatif et une réflexion critique; développer des compétences de communication et de relations interpersonnelles; prendre des décisions et résoudre un problème ; se fixer des buts à atteindre et faire des choix ; s'observer, s'évaluer et se renforcer.

Toutefois, il n'existe pas une seule approche des compétences psychosociales. Ces dernières renvoient à une notion polysémique et polymorphe. Cette difficulté de conceptualisation conduit à un manque de précision des définitions actuellement utilisées en ETP [3, 4], ainsi qu'à une difficulté d'opérationnalisation pour évaluer ces compétences chez les patients [5]. Face à ce constat, d'Ivernois et Gagnayre ont proposé de regrouper les compétences psychosociales en huit catégories en les illustrant par des exemples afin d'aider les équipes à cibler les objectifs pédagogiques de leurs actions d'ETP : informer, éduquer son entourage ; exprimer ses besoins, solliciter l'aide de son entourage ; utiliser les ressources du système de soins, faire valoir ses droits ; analyser des informations reçues sur sa maladie et son traitement ; faire valoir ses choix de santé ; exprimer ses sentiments relatifs à la maladie et mettre en œuvre des conduites d'ajustement; établir des liens entre sa maladie et son histoire de vie ; formuler un projet, le mettre en œuvre [5].

S'il est établi qu'il faut travailler sur les compétences psychosociales dans le domaine de l'ETP, cela n'est pas pour autant facilement réalisable en raison des questions conceptuelles, méthodologiques et opérationnelles qui restent en suspens. L'identification et l'évaluation des compétences psychosociales utiles à renforcer chez les patients afin de les aider à mieux gérer leur maladie et les traitements associés représentent pourtant un enjeu important pour les professionnels qui participent à la conception, à la réalisation et à l'évaluation des interventions éducatives.

Un état des lieux identifiant la façon dont ces compétences psychosociales sont définies et évaluées lors de la mise en œuvre des interventions d'ETP, permettrait d'apporter de nouveaux éléments de réponse. Le diabète de type 1 constitue selon nous un domaine prototypique pour explorer cette question. En effet, le diabète est un champ qui est à la fois pionnier dans le développement de l'ETP et porteur d'enjeux sanitaires partagés avec d'autres types maladies chroniques. Plus précisément, le diabète de type 1 est une pathologie qui commence tôt dans l'existence et qui dure toute la vie. Les diabétiques doivent donc acquérir des compétences d'autosoins et des compétences psychosociales qui seront mobilisées et réactualisées tout au long de leur vie.

Pourtant, l'analyse de la littérature montre que la plupart des revues sur le diabète de type 1 se sont principalement intéressées à l'efficacité des interventions sur la gestion du diabète [6-8]. À notre connaissance, aucune revue n'a porté de façon spécifique sur les compétences psychosociales mises en avant dans le cadre de cette pathologie. Nous avons donc souhaité réaliser une revue de la littérature sur les interventions d'éducation réalisées auprès des diabétiques de type 1 , dont les objectifs sont : 
- évaluer l'évolution de l'importance accordée aux compétences psychosociales dans les interventions en ETP ;

- faire l'inventaire des compétences psychosociales ciblées par ces interventions ;

- indentifier les critères utilisés pour évaluer ces interventions, leur nature (médicale, psychosociale...) et le type de méthode utilisé (qualitatif ou quantitatif) pour les objectiver ;

- repérer parmi ces critères ceux qui permettent d'objectiver des compétences psychosociales telles que définies par l'OMS ;

- élaborer une réflexion conceptuelle problématisant la notion de compétence psychosociale en tant que conduite simultanément individuelle et sociale, à l'interface du psychologique et du social, dont le but est de proposer quelques préconisations opérationnelles pour l'action en ETP.

\section{Méthode}

\section{Sélection des articles}

Les étapes successives de la sélection des articles sont présentées dans la figure 1 . Une revue bibliographique systématique a été réalisée afin de sélectionner les articles qui portaient sur l'évaluation d'une intervention d'éducation du patient (premier critère d'inclusion) à destination d'une population composée uniquement de diabétiques de type 1 (second critère d'inclusion). La recherche s'est déroulée sur les bases de données Medline et PsycINFO avec les mots-clés «diabetes AND type 1 AND patient education ». Ont été retenus les articles en langue anglaise ou française publiés entre janvier 1998 et septembre 2013. La recherche a été limitée à partir de 1998 car il s'agit de l'année où l'OMS a réalisé sa première communication sur les compétences psychosociales.

En raison d'un nombre important d'articles issus de la recherche sur Medline, celle-ci a été restreinte aux articles ayant un résumé disponible et correspondant aux descripteurs majeurs « patient education as topic » et «diabetes mellitus type 1 ». La sélection des articles a été complétée lors de trois étapes de lecture successives : les critères d'inclusion ont été utilisés pour la lecture des titres, pour la lecture des résumés, et enfin pour la lecture de l'ensemble du contenu.

\section{Analyse des articles}

Chaque article retenu a été lu intégralement pour en extraire : (a) des éléments descriptifs généraux (nom des auteurs, année de publication, discipline du premier auteur, population d'étude) ; (b) les critères utilisés pour évaluation ces interventions d'ETP, quels que soient leur nature (médicale, psychosociale) ou leur type (quantitatif, qualitatif) ; (c) les compétences psychosociales mises en avant dans les descriptions des interventions d'éducation. Les compétences recensées ont été classées au sein des catégories et des sous-catégories correspondant à la définition des compétences psychosociales de l'OMS [3].

Après extraction des données, les critères d'évaluation et les compétences psychosociales recensées ont été croisés afin de voir de quelle manière les auteurs objectivent l'acquisition des compétences visées par les interventions d'ETP.

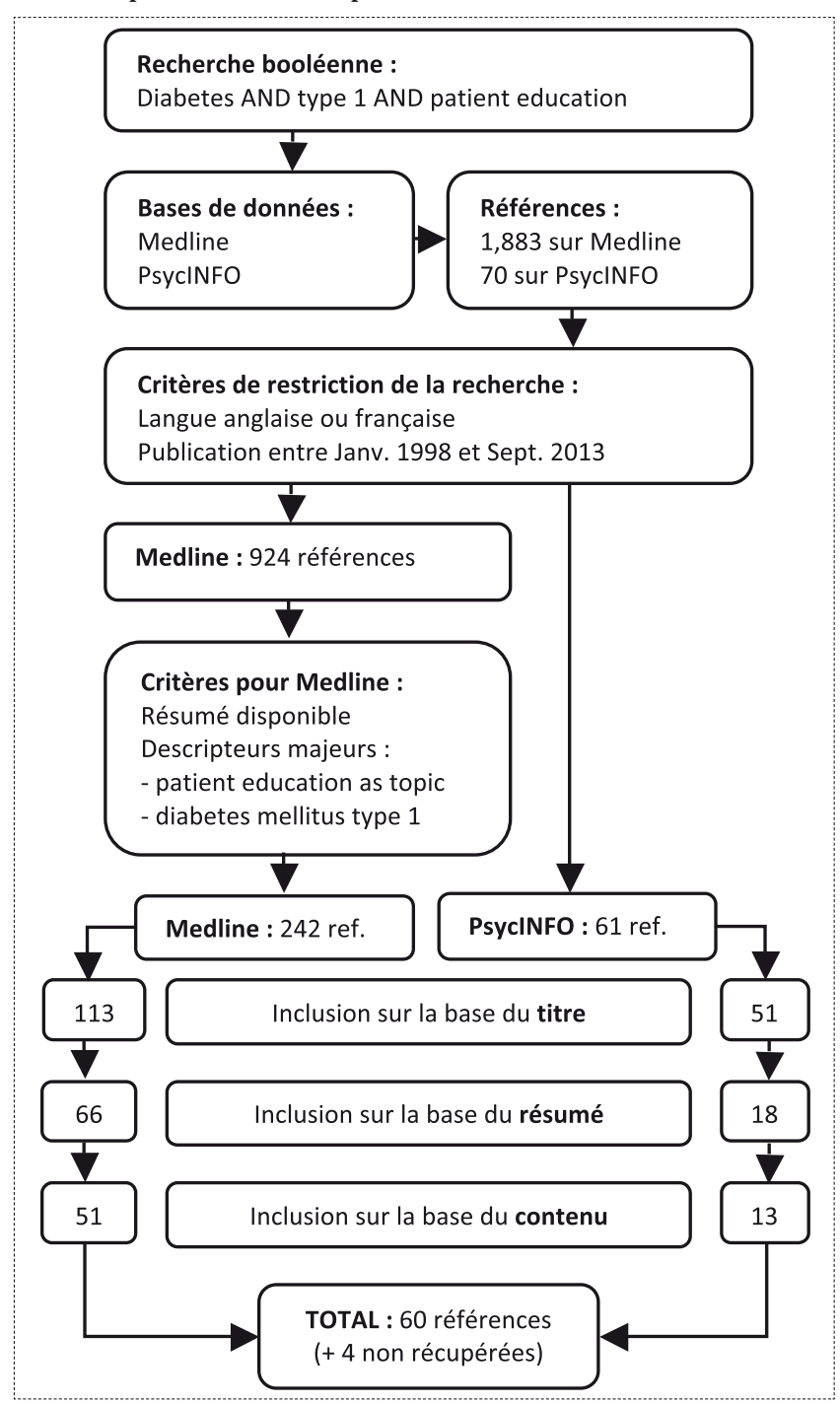

Figure 1 : Stratégie de sélection des articles 


\section{Résultats}

\section{Éléments descriptifs généraux}

Un total de 60 articles a été retenu dans la présente revue. La synthèse des éléments descriptifs est présentée dans le tableau I. La figure 2 montre que la majorité des articles inclus $(86,7 \%)$ a été publiée après 2003 et que le taux d'articles décrivant des compétences psychosociales ciblées par les interventions a été le plus élevé pour les périodes 2006-2007 (40,0 \%) et 2008-2009 (41,7\%). Les premiers auteurs de chaque article s'inscrivent principalement dans les disciplines médicales, telles que l'endocrinologie, la pédiatrie ou la santé publique $(68,3 \%)$. Les autres disciplines les plus représentées sont les sciences humaines et sociales $(21,7 \%)$, notamment la psychologie et les sciences de l'éducation, ainsi que les sciences infirmières $(11,7 \%)$. La discipline d'appartenance du premier auteur est restée inconnue pour deux articles. Les interventions d'ETP ont eu lieu auprès d'une population d'adultes $(61,7 \%)$, d'adolescents $(36,7 \%)$ et d'enfants $(20,0 \%)$.

\section{Compétences psychosociales}

Dans 25 \% des articles, sont présentées les compétences psychosociales visées par les interventions d'ETP. Au total, 23 compétences psychosociales différentes ont été identifiées, dont 43,5 \% renvoient à la communication et aux relations interpersonnelles, 26,1\% à la prise de décision et à l'esprit critique, et 30,4\% à la gestion de soi (tableau II).

\section{Communication et relations interpersonnelles}

Certaines interventions avaient pour objectif d'aider les patients à acquérir des compétences liées à la communication. Il s'agissait d'apprendre à identifier les différents styles de communication et de connaître leurs effets potentiels dans la relation avec autrui, d'apprendre à gérer les moments difficiles ou gênants lors des discussions à propos de leur maladie, et de prendre l'habitude de s'exprimer face à autrui [27]. Il s'agissait également d'apprendre à s'exprimer sur ses propres difficultés ainsi que sur les besoins et les émotions liées à la maladie et à l'autogestion [48]. Afin d'améliorer les relations des patients avec autrui, une intervention visait également à développer des

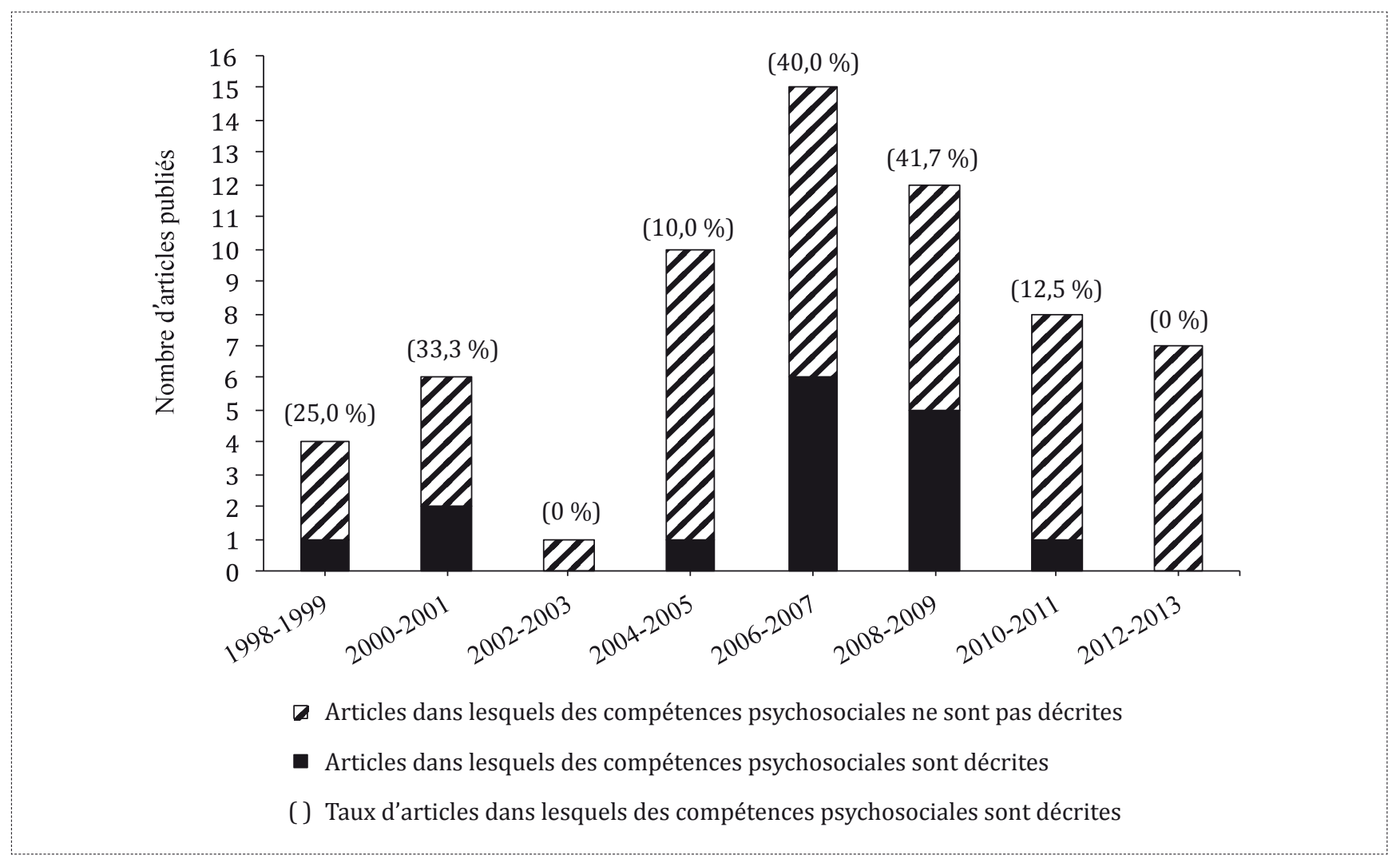

Figure 2 : Nombre d'articles publiés entre 1998 et 2013 
Tableau I : Éléments descriptifs généraux, méthode et critères d'évaluation

\begin{tabular}{|c|c|c|c|}
\hline Référence & Discipline & Méthode & Critères d'évaluation \\
\hline Aguilar 2012 [9] & $\begin{array}{l}\text { Sciences } \\
\text { infirmières }\end{array}$ & Quanti. & Glycémie, fréquence de contrôles glycémiques \\
\hline Amsberg 2009 [10] & Médecine & Quanti. & $\begin{array}{l}\text { Comportements d'autogestion, stress perçu, anxiété, dépression, bien-être psychologique, } \\
\text { peur de l'hypoglycémie, émotions liées au diabète }\end{array}$ \\
\hline Araszkiewicz 2008 [11] & Médecine & Quanti. & $\begin{array}{l}\text { Glycémie, connaissances sur la maladie et l'autogestion, qualité de vie, attitude envers } \\
\text { le diabète et l'autogestion, satisfaction du traitement }\end{array}$ \\
\hline Attari 2006 [12] & Médecine & Quanti. & Glycémie, style de coping \\
\hline Benhamou 2010 [13] & Médecine & Quanti. & $\begin{array}{l}\text { Glycémie, indice de masse corporelle, nombre d'hypoglycémie, quantité d'insuline, } \\
\text { fréquence de contrôle glycémique }\end{array}$ \\
\hline Booker $2008[14]$ & Psychologie & Quali. & $\begin{array}{l}\text { Vécu de l'ETP, changements apportés à l'autogestion, perception des éléments importants } \\
\text { de l'éducation }\end{array}$ \\
\hline Bruttomesso 2006 [15] & Médecine & Quanti. & Connaissances sur la maladie et sur l'autogestion \\
\hline Cabrera $2013[16]$ & Médecine & Quanti. & Glycémie \\
\hline Couper 1999 [17] & Médecine & Quanti. & Glycémie, connaissances sur la maladie et l'autogestion \\
\hline Cox $2001[18]$ & Médecine & Quanti. & $\begin{array}{l}\text { Glycémie, connaissances sur la maladie et l'autogestion, conséquences médicales } \\
\text { négatives lieés au diabète, dépression, qualité de vie, conflits familiaux }\end{array}$ \\
\hline Cox $2004[19]$ & Médecine & Quanti. & Glycémie, nombre d'hypoglycémie, capacité d'estimer le taux de glucose dans le sang \\
\hline Cox $2008[20]$ & Médecine & Quanti. & $\begin{array}{l}\text { Décision de ne pas rouler lorsque le taux de glucose est trop faible, décision de manger } \\
\text { des glucides à action rapide }\end{array}$ \\
\hline Dinneen 2009 [21] & Médecine & Quanti. & $\begin{array}{l}\text { Glycémie, nombre d'hypoglycémie, qualité de vie, émotions liées au diabète, anxiété, } \\
\text { dépression }\end{array}$ \\
\hline Forlani 2006 [22] & Médecine & Quanti. & Bien-être psychologique, qualité de vie \\
\hline Fritsche 1998 [23] & Médecine & Quanti. & Glycémie \\
\hline García-Pérez 2010 [24] & Médecine & Quanti. & $\begin{array}{l}\text { Glycémie, indice de masse corporelle, nombre d'hospitalisation causée par le diabète, } \\
\text { connaissances sur la maladie et l'autogestion, anxiété, style de coping }\end{array}$ \\
\hline George 2008 [25] & Médecine & Quanti. & $\begin{array}{l}\text { Glycémie, indice de masse corporelle, nombre d'hypoglycémie, quantité d'insuline, } \\
\text { connaissances sur la maladie et l'autogestion, comportements d'autogestion, qualité } \\
\text { de vie, sentiment d'auto-efficacité, peur de l'hypoglycémie, croyances sur la maladie }\end{array}$ \\
\hline Grey $1999[26]$ & $\begin{array}{l}\text { Sciences } \\
\text { infirmières }\end{array}$ & Quanti. & Glycémie, sentiment d'auto-efficacité, dépression, qualité de vie, style de coping \\
\hline Grey 2009 [27] & $\begin{array}{l}\text { Sciences } \\
\text { infirmières }\end{array}$ & Quanti. & $\begin{array}{l}\text { Glycémie, sentiment d'auto-efficacité, dépression, qualité de vie, style de coping, } \\
\text { soutien social }\end{array}$ \\
\hline Hains $2000[28]$ & Psychologie & Quanti. & Glycémie, anxiété, stress perçu \\
\hline Hermanns 2007 [29] & Médecine & Quanti. & $\begin{array}{l}\text { Glycémie, nombre d'hypoglycémie, symtômes d'hypoglycémie, qualité de vie, } \\
\text { émotions liées au diabète, anxiété, dépression }\end{array}$ \\
\hline Hernandez 2004 [30] & $\begin{array}{l}\text { Sciences } \\
\text { infirmières }\end{array}$ & Quanti. & Glycémie, comportements d'autogestion, qualité de vie, satisfaction de l'intervention \\
\hline Hernandez $2008[31]$ & $\begin{array}{l}\text { Sciences } \\
\text { infirmières }\end{array}$ & Quanti. & Comportements d'autogestion, qualité de vie \\
\hline Hopkins 2012 [32] & Médecine & Quanti. & $\begin{array}{l}\text { Glycémie, nombre d'hypoglycémie, anxiété, dépression, émotions liées au diabète, } \\
\text { bien-être psychologique }\end{array}$ \\
\hline
\end{tabular}


Tableau I : Éléments descriptifs généraux, méthode et critères d'évaluation (suite)

\begin{tabular}{|c|c|c|c|}
\hline Référence & Discipline & Méthode & Critères d'évaluation \\
\hline Howe 2005 [33] & Médecine & Quanti. & $\begin{array}{l}\text { Glycémie, connaissances sur la maladie et l'autogestion, adhérence aux traitements, } \\
\text { coopération parents-enfants }\end{array}$ \\
\hline Howorka $2000[34]$ & Médecine & Quanti. & Satisfaction du traitement, croyances (santé, maladie), contrôle perçu \\
\hline Jansà 2006 [35] & Médecine & Quanti. & $\begin{array}{l}\text { Glycémie, nombre d'hypoglycémie, fréquence de contrôle glyémique, connaissances } \\
\text { sur la maladie et l'autogestion, qualité de vie }\end{array}$ \\
\hline Kinsley 1999 [36] & Médecine & Quanti. & Glycémie, capacité d'estimer le taux de glycémie dans le sang, humeur \\
\hline Kubiak 2006 [37] & Psychologie & Quanti. & $\begin{array}{l}\text { Glycémie, capacité d'estimer le taux de glycémie dans le sang, dépression, anxiété, } \\
\text { croyances sur la santé }\end{array}$ \\
\hline Lagger 2010 [38] & Médecine & Mixte & $\begin{array}{l}\text { Motivation à apprendre et à prendre soin de soi-même, attitudes et comportements liés } \\
\text { à la santé, qualité de la relation soignant-soigné }\end{array}$ \\
\hline Lawson 2005 [39] & Médecine & Quanti. & Quantité d'insuline consommée, compliance, qualité de vie, fonctionnement familial \\
\hline Lemozy-Cadroy 2002 [40] & Médecine & Quanti. & $\begin{array}{l}\text { Glycémie, connaissances sur la maladie et l'autogestion et compétences } \\
\text { dans l'autogestion }\end{array}$ \\
\hline Liesenfeld 2000 [41] & Médecine & Quanti. & $\begin{array}{l}\text { Glycémie, nombre d'hypoglycémie, fréquence d'injections d'insuline, } \\
\text { effets indésirables du diabète }\end{array}$ \\
\hline Mannucci 2005 [42] & Médecine & Quanti. & Glycémie, bien-être psychologique \\
\hline Müller 2013 [43] & Médecine & Quanti. & $\begin{array}{l}\text { Glycémie, nombre d'hypoglycémie et d'acidocétose, satisfaction du traitement, qualité } \\
\text { de vie }\end{array}$ \\
\hline Nansel 2007 [44] & Médecine & Quanti. & $\begin{array}{l}\text { Glycémie, adhérence au traitement, sentiment d'auto-efficacité, qualité de vie, satisfaction } \\
\text { de l'intervention }\end{array}$ \\
\hline Nicholas 2012 [45] & Travail social & Mixte & Soutien social, effets perçus de l'intervention, obstacles à la participation, bénéfices perçus \\
\hline Nordfeldt 2005 [46] & Médecine & Quanti. & Glycémie, nombre d'hypoglycémie \\
\hline Nunn 2006 [47] & NR & Quanti. & Glycémie, connaissances sur la maladie et l'autogestion \\
\hline Pélicand 2006 [48] & Médecine & Quanti. & Connaissances sur la maladie et l'autogestion, comportements d'autogestion \\
\hline Piana 2010 [49] & Médecine & Mixte & $\begin{array}{l}\text { Effets de l'intervention sur le souci accordé à l'autogestion et au bien-être, la conscience } \\
\text { de soi }\end{array}$ \\
\hline Plank 2004 [50] & Médecine & Quanti. & $\begin{array}{l}\text { Glycémie, quantité d'insuline/jour, nombre d'injections d'insuline/jour, connaissances } \\
\text { sur la maladie et l'autogestion, indice de masse corporelle }\end{array}$ \\
\hline Povlsen 2005 [51] & $\begin{array}{l}\text { Santé } \\
\text { publique }\end{array}$ & Quanti. & Glycémie, connaissances sur la maladie et l'autogestion \\
\hline Povlsen 2008 [52] & $\begin{array}{l}\text { Santé } \\
\text { publique }\end{array}$ & Mixte & $\begin{array}{l}\text { Glycémie, perception du moment du diagnostic d'éducation thérapeutique, soutien fourni } \\
\text { par les professionnels, soutien et relations entretenus avec la famille et les amis, } \\
\text { vie présente et vie future avec la maladie }\end{array}$ \\
\hline Qayyum 2010 [53] & Médecine & Quanti. & Glycémie, indice de masse corporelle, régime d'insuline \\
\hline Rankin 2012 [54] & $\begin{array}{l}\text { Sciences de } \\
\text { l'éducation }\end{array}$ & Quanti. & Glycémie \\
\hline Santiprabhob 2008 [55] & Médecine & Quanti. & Glycémie \\
\hline Schachinger 2005 [56] & Médecine & Quanti. & $\begin{array}{l}\text { Glycémie, nombre d'hypoglycémie, peur de l'hypoglycémie, qualité de vie, bien-être } \\
\text { psychologique, humeur, contrôle perçu }\end{array}$ \\
\hline Smith 2006 [57] & $\begin{array}{l}\text { Sciences de } \\
\text { l'éducation }\end{array}$ & Mixte & Glycémie, vécu de l'autogestion au quotidien \\
\hline
\end{tabular}


Tableau I : Éléments descriptifs généraux, méthode et critères d'évaluation (suite)

\begin{tabular}{|c|c|c|c|}
\hline Référence & Discipline & Méthode & Critères d'évaluation \\
\hline Snoek 2001 [58] & Psychologie & Quanti. & $\begin{array}{l}\text { Comportements d'autogestion, symptômes psychopathologiques, } \\
\text { bien-être psychologique, émotions liées au diabète, peur de l'hypoglycémie, } \\
\text { obstacles perçus dans l'autogestion }\end{array}$ \\
\hline Speight 2010 [59] & Psychologie & Quanti. & Glycémie, qualité de vie, satisfaction du traitement \\
\hline Verbeek 2011 [60] & NR & Quanti. & Glycémie \\
\hline Viklund 2007a [61] & Médecine & Quanti. & $\begin{array}{l}\text { Glycémie, connaissances sur la maladie et l'autogestion, perturbations engendrées } \\
\text { par le diabète, attitude envers le diabète et l'autogestion, sentiment d'indépendance }\end{array}$ \\
\hline Viklund 2007b [62] & Médecine & Quanti. & Glycémie, empowerment \\
\hline von Sengbush 2006 [63] & Médecine & Quanti. & $\begin{array}{l}\text { Connaissances sur la maladie et l'autogestion, qualité de vie, satisfaction } \\
\text { de l'intervention }\end{array}$ \\
\hline Waller 2008 [64] & Psychologie & Mixte & $\begin{array}{l}\text { Glycémie, nombre d'hypoglycémie, indice de masse corporelle, qualité de vie, } \\
\text { satisfaction du traitement, sentiment d'auto-efficacité, responsabilité dans l'autogestion, } \\
\text { conflits familiaux liés au diabète, attentes, inquiétudes face à intervention, avantages } \\
\text { et inconvénients perçus après intervention }\end{array}$ \\
\hline Wdowik 2000 [65] & Nutrition & Quanti. & $\begin{array}{l}\text { Glycémie, comportements et connaissances sur la maladie, attitude envers le diabète } \\
\text { et l'autogestion }\end{array}$ \\
\hline Whittemore 2010 [66] & $\begin{array}{l}\text { Sciences } \\
\text { infirmières }\end{array}$ & Quanti. & $\begin{array}{l}\text { Glycémie, qualité de vie, stress perçu, style de coping, sentiment d'auto-efficacité, } \\
\text { dépression }\end{array}$ \\
\hline Whittemore 2012 [67] & $\begin{array}{l}\text { Sciences } \\
\text { infirmières }\end{array}$ & Quanti. & $\begin{array}{l}\text { Glycémie, comportements d'autogestion, qualité de vie, stress perçu, style de coping, } \\
\text { auto-efficacité, conflits familiaux, dépression }\end{array}$ \\
\hline Zoffmann 2006 [68] & $\begin{array}{l}\text { Santé } \\
\text { publique }\end{array}$ & Quanti. & $\begin{array}{l}\text { Comportements d'autogestion, émotions liées au diabète, compétences perçues } \\
\text { dans l'autogestion }\end{array}$ \\
\hline
\end{tabular}

Note : les critères d'évaluation médicaux sont en gras; les critères d'évaluation psychosociaux sont en italique.

compétences permettant de surmonter les sentiments de honte et de crainte d'être rejeté qui sont générés par la maladie [49].

La gestion du diabète implique des restrictions dans certaines activités quotidiennes réalisées en groupe. Il est alors estimé que les patients doivent acquérir des compétences, soit pour décliner certaines propositions du groupe avec diplomatie, soit pour entreprendre des négociations et trouver des compromis. Pour surmonter ces difficultés, une intervention visait à apprendre aux patients à résister à l'influence des pairs et à résoudre les conflits qui peuvent émerger d'un refus éventuel (notamment pour la consommation de drogue, d'alcool ou de certains aliments) [26]. Une autre intervention avait pour objectif d'aider les patients à connaître différentes sources de conflits en communication afin de savoir comment les gérer le plus positivement possible [27].

Les difficultés suscitées par la gestion du diabète impliquent également d'acquérir des compétences permettant de rechercher du soutien social et d'éduquer son entourage sur la maladie. La recherche du soutien auprès d'autres diabétiques devait permettre aux patients : d'améliorer la confiance en soi, de mieux accepter la maladie et d'avoir des sentiments plus positifs [49] ; mais également de réduire le sentiment d'isolement et de partager des idées sur la façon de vivre au quotidien la maladie [55]. L'éducation de l'entourage familial par les patients devait permettre de les amener à devenir plus compréhensifs face à la maladie et aux difficultés d'autogestion [57]. D'autre part, une intervention avait pour objectif d'aider les patients à savoir communiquer avec les professionnels de santé au bon moment lorsqu'il s'agit d'obtenir une assistance face aux difficultés [14].

\section{Prise de décision et esprit critique}

Plusieurs interventions ont mis en avant la nécessité d'acquérir des compétences liées à la prise de décision et à la résolution de problèmes pour une autogestion efficace du diabète. Ainsi, certains patients devaient 
Tableau II : Catégorisation des compétences psychosociales relevées dans la description des interventions en fonction de la définition des CPS proposée par l'OMS

\section{Communication et relation interpersonnelle}

Compétences de communication

interpersonnelle :

- Identifier les différents styles de communication (agressif, autoritaire, passif) et connaître leurs effets [27].

- Gérer les moments difficiles ou gênants dans les discussions sur sa propre maladie [27].

- Prendre l'habitude de s'exprimer sur les difficultés, les besoins et les émotions ressenties liées au diabète [48].

- Surmonter la honte de son diabète et la crainte de ne pas être accepté par autrui [49].

Compétences de refus ou de négociation :

- Résoudre les conflits en identifiant les différentes sources de conflits (évitement, confrontation, humour) [27].

- Résister à l'influence des pairs (drogue, alcool, alimentation) et savoir résoudre les conflits qui peuvent émerger [26].

Compétences de recherche de soutien social et d'éducation :

- Communiquer avec les professionnels de santé au bon moment pour obtenir assistance face aux difficultés [14].

- Rechercher l'appui d'autres diabétiques pour : réduire le sentiment d'isolement, partager informations et idées sur la façon de vivre sa maladie [55].

- Mieux accepter la maladie, améliorer sa confiance en soi et envers ses capacités [49].

- Éduquer et informer son entourage pour les amener à devenir plus compréhensifs face à la maladie et face aux difficultés quotidiennes dans l'autogestion [57].

Prise de décision et esprit critique

Compétences de prise de décision et de résolution de problème :

- Acquérir de l'autonomie et des responsabilités pour les décisions prises dans l'autogestion de la maladie [55].

- Exprimer, poser et évaluer les difficultés quotidiennes liées au diabète [68].

- Définir les problèmes et leurs conséquences pour prendre des décisions adaptées [28].

Compétences d'esprit critique :

- Identifier les situations sociales problématiques (influence des pairs, pression sociale) pouvant nuire à la gestion du diabète pour trouver des solutions [57].

- Identifier les émotions et les pensées négatives (exagérations, attentes irréalistes, réactions excessives) et connaître leurs effets sur le comportement et la gestion du stress $[27,28,58]$.

- Contester et restructurer les pensées négatives en se basant sur les faits et en développant une réflexion plus réaliste de la situation [28].
Gestion de soi

Compétences pour assumer le contrôle ou amorcer une transformation :

- Trouver des sources de motivation et des moyens de développer l'estime de soi [55].

- Etre à l'écoute de son corps pour reconnaître les signaux corporels qui annoncent une glycémie faible ou élevée [30,31].

- Définir des objectifs à atteindre en fonction de ses besoins et évaluer les progrès réalisés [44].

- Connaître ses propres contraintes dans la gestion de sa maladie [12].

Compétences pour la gestion du stress et des émotions liées au diabète :

- Faire de la relaxation pour diminuer le stress causé par le diabète $[12,27]$ et la peur de I'hypoglycémie [10].

- Gérer l'impact psychologique et émotionnel causé par des difficultés de l'autogestion [14].

- Connaître les causes extérieures à soi qui génèrent le stress [12].

Note : les catégories en gras correspondent aux compétences psychosociales proposées par l'OMS [3] ; les termes listés sous chaque catégorie correspondent aux compétences relevées dans les articles étudiés.

apprendre à exprimer, poser et évaluer les difficultés quotidiennes liées au diabète pour les résoudre plus efficacement, obtenir un meilleur contrôle glycémique, des compétences perçues plus élevées dans l'autogestion, et moins de complications liées au diabète [68]. D’autres patients devaient apprendre à définir clairement les problèmes et leurs conséquences afin de prendre les décisions les plus adaptées face aux difficultés [28]. Concernant les enfants et les adolescents diabétiques en particulier, une intervention visait à développer l'autonomie et la responsabilité dans les décisions prises pour l'autogestion [55].
En outre, les patients peuvent acquérir des compétences leur permettant de faire preuve d'un esprit critique. Trois interventions visaient à apprendre aux patients à savoir identifier les pensées qui entravent les comportements d'autosoins et la gestion du stress $[27,28,58]$. Cela notamment afin d'apprendre à contester et à restructurer les pensées négatives en se basant sur les faits et en développant une réflexion plus réaliste de la situation [28]. Enfin, une intervention visait à apprendre aux patients à identifier les situations sociales problématiques (influence des pairs, pression sociale) qui peuvent nuire à l'autogestion afin de se montrer critique et de trouver des solutions adaptées [57]. 


\section{Gestion de soi}

Des interventions visaient également à apprendre aux patients à assumer le contrôle de la maladie ou à amorcer une transformation dans l'autogestion. Plus précisément, il était question d'apprendre à définir des objectifs à atteindre en fonction de ses propres besoins, et de s'auto-évaluer efficacement dans les progrès réalisés [44], ou bien encore d'apprendre à trouver des sources de motivation pour amener des changements et à rechercher des moyens permettant de développer l'estime de soi [55]. En outre, deux interventions ont visé des compétences liées à la conscience de soi : être à l'écoute de son propre corps pour reconnaître les signaux corporels qui annoncent une glycémie faible ou élevée et pour permettre de connaître les normes de son propre corps dans les différents moments de la journée et dans les différentes saisons de l'année [30, 31]. Par ailleurs, une autre compétence liée au contrôle de la maladie renvoyait au fait de prendre conscience de ses contraintes personnelles pour tendre vers une autogestion efficace [12].

Un dernier type de compétences visées dans les interventions concernait la gestion du stress et des émotions dues à la maladie. Pour ce faire, trois interventions visaient à apprendre aux patients à effectuer des séances de relaxation pour gérer le stress et la peur de l'hypoglycémie $[10,12,27]$. Cet apprentissage était justifié dans l'une des interventions par le fait que la relaxation permettrait de diminuer les comportements d'évitement face aux difficultés d'autogestion [10]. En complémentarité des compétences liées à la relaxation, l'une de ces interventions visait à aider les patients à savoir déterminer les causes environnementales de leur propre stress [12]. Enfin, une intervention visait à apprendre aux patients à gérer l'impact psychologique et émotionnel que génère l'autogestion [14].

\section{Nature des critères et méthodes d'évaluation}

Une liste des critères utilisés pour chaque étude est présentée dans le tableau I. Ils se différencient tant par leur nature (médicale ou psychosociale) que par leurs méthodes d'évaluation (qualitative ou quantitative). La grande majorité des études ont utilisé une méthode d'évaluation uniquement quantitative (88,3\%). À l'exception d'un seul cas, les études qui utilisent une méthode d'évaluation qualitative recourent également à une méthode d'évaluation quantitative.

\section{Méthode d'évaluation qualitative}

Les critères explorés par la méthode d'évaluation qualitative se réfèrent à des données subjectivées recueillies au moyen d'entretiens semi-directifs visant à connaître le vécu lié à la maladie, à la participation aux interventions d'ETP, et aux relations entretenues avec autrui. Plus précisément, les patients ont été amenés à s'exprimer sur les thématiques suivantes : les changements apportés par l'intervention d'ETP et ses bénéfices sur l'autogestion [14, 45, 49, $52,64]$; les éléments que les patients percevaient comme importants dans l'ETP [14]; les obstacles liés au choix de participer à l'ETP et le souci accordé à l'autosoin et au bienêtre [49]; la motivation à apprendre et à prendre soin de soi-même, les attitudes et les comportements liés à la santé [38] ; la qualité des relations soignant-soigné [38, 45] ; le soutien fourni par les professionnels, la famille et les amis $[45,52]$. Certaines études ont procédé de manière différente, en demandant aux patients de s'exprimer sur leurs propres données médicales ainsi que sur des photos illustrant différentes facettes de l'autogestion (ces derniers avaient pour charge de prendre régulièrement des photos de leur propre quotidien en rapport avec l'autogestion) [57].

\section{Méthode d'évaluation quantitative}

La méthode d'évaluation quantitative renvoie à l'exploration de critères de nature médicale pour la majorité des études (73,3\%). Pour ces études, le taux d'hémoglobine glyquée (HbA1c) a été systématiquement utilisé pour juger de l'équilibre glycémique. D'autres données médicales ont parfois été associées en complément, comme le nombre d'épisodes d'hypoglycémie survenue sur une période donnée $(20,0 \%)$, l'indice de masse corporelle $(10,0 \%)$, la quantité d'insuline consommée par jour (5,0\%), et les effets indésirables liés au diabète $(5,0 \%)$.

Les résultats des interventions d'ETP ont également été documentés à travers la mesure des connaissances (26,7\%) et des comportements $(23,3 \%)$ liés à la maladie et à l'autogestion. Les connaissances ont généralement été évaluées au moyen de questions à choix multiples sur les thématiques suivantes : causes du diabète, comportements préconisés à adopter face à l'hypoglycémie et à l'hyperglycémie, alimentation, soin des pieds, etc. Les comportements sont généralement évalués à travers la fréquence de réalisation de certains comportements au cours d'une période donnée, tels que par exemple : les injections d'insuline, le contrôle de glucose dans le sang, les exercices physiques, le respect des recommandations diététiques et des prescriptions médicamenteuses. 
Des critères de nature psychosociale ont également été utilisés pour l'évaluation quantitative des interventions. Les critères les plus fréquemment observés dans les articles étaient : la qualité de vie $(33,3 \%)$, la dépression $(16,7 \%)$, les émotions (15\%), et plus précisément la charge émotionnelle liée à l'autogestion, l'humeur et la peur de l'hypoglycémie, l'anxiété (11,7\%), et le sentiment d'auto-efficacité $(11,7 \%)$. Suivaient ensuite le coping $(10,0 \%)$, le bien-être psychologique, la satisfaction du traitement $(8,3 \%)$ et de l'intervention (6,7\%), et le stress perçu $(6,7 \%)$. Enfin, parmi les critères les moins souvent utilisés, se trouvaient : l'attitude à l'égard du diabète ou de l'autogestion $(5,0 \%)$, les croyances sur la santé et sur la maladie $(5,0 \%)$, le soutien social perçu (3,3\%), le contrôle perçu sur la maladie $(3,3 \%)$ et l'adhérence au traitement $(3,3 \%)$.

\section{Évaluation des compétences psychosociales}

Parmi les articles qui ont mis en avant des compétences psychosociales, un seul semble avoir eu recours à une méthode d'évaluation quantitative permettant de mesurer ces compétences acquises par les patients. Il s'agissait d'une intervention visant à apprendre aux patients la relaxation afin de diminuer les comportements d'évitement dans l'autogestion, d'agir sur le stress causé par la maladie, et de diminuer la peur liée à l'hypoglycémie [10] ; des échelles de mesure ont été utilisées pour objectiver l'acquisition de ces compétences.

Concernant les articles qui ont eu recours à une méthode d'évaluation qualitative, trois d'entre eux ont également précisé les compétences psychosociales visées par leur intervention [14, 49,57]. L'évaluation a été réalisée à l'aide d'entretiens semi-directifs invitant les patients à s'exprimer sur certaines thématiques. Toutefois, la description des thématiques abordées dans ces entretiens n'était pas suffisamment précise pour savoir si elles permettaient d'évaluer ces compétences psychosociales.

\section{Discussion}

\section{Des compétences psychosociales peu explicitées et peu évaluées}

Le principal constat de notre revue concerne la difficulté d'identifier les compétences psychosociales : rares sont les auteurs qui explicitent clairement les compétences visées dans les interventions d'ETP. En effet, dans la majorité des articles, les objectifs pédagogiques en termes de compétences psychosociales ne sont pas mentionnés. Cela est peut-être dû au fait que les interventions ne sont pas suffisamment décrites par les auteurs : ces dernières semblent davantage décrites à travers les activités proposées au patient qu'à travers les compétences sociales que ces activités sont censées développer. Cette observation est confirmée par une revue de la littérature qui montre que les données méthodologiques renvoyant à la description et à la mise en place des interventions d'éducation thérapeutique évaluées sont souvent sommaires [8]. Pourtant, le nombre d'articles explicitant les compétences psychosociales visées dans les interventions a connu une augmentation soudaine de publications à partir de 2006, jusqu'à connaître une nette régression depuis 2010. Cette fluctuation de l'intérêt des auteurs pour décrire les compétences psychosociales dans la description méthodologique de leur intervention reste actuellement difficile à interpréter.

En outre, la quasi-totalité des articles n'a pas utilisé de critères permettant d'objectiver l'acquisition des compétences psychosociales visées dans les interventions d'ETP. Ces résultats vont dans le sens des observations déjà faites : les compétences psychosociales sont rarement formulées et mesurées dans l'évaluation des interventions d'ETP [5]. Pourtant, l'évaluation des compétences acquises par les patients constitue un enjeu important pour objectiver l'efficacité de ces interventions [69]. Toutefois, le fait que les compétences psychosociales ne soient pas explicitées dans la description de certaines interventions ne signifie pas pour autant que ces compétences ne sont pas développées chez les patients. Par exemple, un certain nombre d'articles inclus dans notre revue ont utilisé une échelle de coping pour mesurer la capacité de se centrer sur le problème avec une approche active et systématique. D'autres encore ont utilisé une échelle de sentiment d'auto-efficacité pour mesurer la croyance qu'ont les patients dans leurs capacités à mobiliser des ressources permettant de maîtriser les différentes situations de l'autogestion.

Enfin, nous avons constaté que peu d'articles ont eu recours à une méthode d'évaluation qualitative. Ce type de méthode présente pourtant un intérêt dans l'évaluation des interventions d'ETP. La démarche qualitative implique une approche compréhensive, contextualisée et holistique des phénomènes in vivo [70]. Dans notre cas, elle offre la possibilité d'appréhender les compétences psychosociales que les patients nécessitent, acquièrent et mobilisent, en tenant compte de la situation vécue et de la complexité des processus en jeu dans l'adaptation à la maladie et aux traitements. Outre les questionnaires habituellement utilisés, l'évaluation des interventions devrait alors intégrer l'usage 
des techniques qualitatives, telles que les entretiens semidirectifs et les récits de vie [5]. Cette stratégie méthodologique permettrait de considérer de façon plus fine l'expérience subjective de la maladie (e.g. sens donné, étiologie, «maladie du malade») et de contribuer ainsi à une problématisation opérationnelle des compétences psychosociales en ETP (repérage, action éducative, évaluation) en fonction des spécificités des situations rencontrées et des patients.

\section{Un fort accent mis sur les compétences d'autosoin}

Selon nous, la place relative accordée aux compétences psychosociales peut trouver deux explications. Premièrement, les interventions d'éducation thérapeutique semblent avoir été longtemps élaborées à travers une approche biomédicale de l'éducation. Une revue de la littérature montre en effet que les interventions mettent davantage l'accent sur des résultats physiologiques et sur le respect des instructions d'autosoin que sur des variables psychologiques [71]. Notre revue semble concorder avec ces résultats : les données médicales, le respect des recommandations des professionnels concernant les comportements de santé, et les connaissances liées à la maladie et à son autogestion font partie des critères les plus souvent mis en avant pour évaluer les interventions d'ETP. En revanche, mise à part la qualité de vie, les critères de nature psychosociale sont relativement moins utilisés pour l'évaluation. Ces constatations peuvent également être expliquées par le fait que la majorité des premiers auteurs de nos articles sont rattachés à la discipline médicale.

Deuxièmement, les compétences psychosociales identifiées dans notre revue semblent difficilement opérationnalisables, comme cela a déjà été souligné [5], alors que l'objectivation des compétences d'autosoin acquises par les patients semble plus facile à mettre en œuvre par les auteurs. Pour les professionnels de santé développant l'éducation thérapeutique, l'évaluation des compétences d'autosoin est plus proche de leur champ disciplinaire et de leur pratique courante, que l'évaluation des compétences psychosociales. En effet, la formation initiale de ces professionnels de santé ne les prépare pas à repérer les compétences psychosociales, les travailler et les évaluer. D’où peut-être la nécessité de leur proposer des formations plus adaptées à la pratique éducative et/ou de s'associer à des professionnels issus des Sciences Humaines et Sociales qui disposent des connaissances et des savoirfaire pour concevoir des dispositifs d'évaluation pluridisciplinaires.
Dans cette perspective, la littérature francophone rapporte quelques expériences de terrain qui soulignent l'intérêt du psychologue dans les différentes étapes de la démarche éducative, notamment pour évaluer et renforcer les compétences psychosociales [72, 73]. Ces professionnels nous semblent être des partenaires d'autant plus intéressants que la notion-même de compétence psychosociale a émergé et s'est développée dans le domaine de la psychologie et que la question de son opérationnalisation a toujours constitué une préoccupation pour les chercheurs de cette discipline [1].

\section{Limites de l'étude}

Pour des raisons pratiques de faisabilité, la revue a été restreinte à deux bases de données et à un champ pathologique. Toutefois, il nous semble que les compétences psychosociales que nous avons identifiées ne sont pas spécifiques au diabète de type 1 et qu'elles pourraient être pertinentes à développer chez des patients atteints d'autres types de pathologies. D'autre part, le travail d'identification des compétences psychosociales à partir des articles sélectionnés a nécessité une interprétation de notre part. Comme nous l'avons déjà noté, les compétences décrites dans la littérature n'étaient pas toujours identifiables de façon claire. De plus, la définition de l'OMS sur laquelle nous nous sommes appuyés ne facilite pas l'identification directe de ces compétences. Encore aujourd'hui, ces compétences restent définies de façon peu précise, ce qui renforce le risque qu'elles soient utilisées comme un concept fourretout par un certain nombre de professionnels.

Enfin, le choix des bases de données constitue également une limite. Celles-ci référencent des revues qui répondent à un standard scientifique international (e.g. valorisation de l'essai contrôlé randomisé) qui non seulement ne couvre pas tout le champ de ce qui se fait en termes d'évaluation des compétences psychosociales, mais aussi se révèle peu adapté à la nature du phénomène évalué. Des méthodes innovantes ou des expériences de terrain peuvent donc ne pas avoir été publiées dans ce type de base. Pour les recenser, il pourrait être intéressant d'accéder à la littérature « grise » émanant des équipes éducatives elles-mêmes.

\section{Implications : enjeux d'une approche psychosociale}

Les interventions éducatives sont aujourd'hui pensées comme des interventions complexes. Ces dernières sont caractérisées par une multiplicité de composantes en 
interaction (e.g. nature des pratiques pédagogiques, attentes des professionnels, environnement social des patients, spécificités de la pathologie, etc.). Leur évaluation nécessite d'adopter des approches originales pour rendre compte de la complexité des systèmes de santé. L'évaluation de ces interventions complexes exige donc le recours à des modèles d'évaluation flexibles et adaptés aux contextes de leur mise en œuvre [74].

La psychologie sociale constitue l'une des disciplines des Sciences Humaines et Sociales qui dispose d'outils théoriques et méthodologiques pertinents pour répondre aux problématiques soulevées autour des compétences psychosociales dans le champ de l'ETP. Elle étudie le rapport que les individus entretiennent avec la maladie et les comportements de santé, en tenant compte du contexte social, c'est-à-dire le « système social dans lequel est enraciné un comportement (valeurs, normes, représentations sociales) ainsi que les relations entretenues par les individus avec autrui » [75]. Cette discipline pose un regard psychosocial [76] qui permet de contextualiser les comportements de santé dans et par les faits d'inscription et de participation sociales. Sa spécificité réside dans la lecture ternaire portée sur les phénomènes, à travers l'articulation dynamique et relationnelle de trois entités : Sujet-Alter-Objet. Appliqué à la situation d'une maladie chronique (figure 3), ce regard permettrait de comprendre l'articulation qui s'établit entre le patient (Sujet), les comportements dans l'ETP (Objet), et les autres (Alter), ces derniers pouvant être des professionnels de santé, des proches, des patients, ou des groupes sociaux d'appartenance.

La conceptualisation des compétences psychosociales dans l'optique dynamique et relationnelle de ce regard reste toujours un enjeu aujourd'hui. L'intérêt actuel porté à ces compétences est essentiellement centré sur les aspects individuels des comportements de santé (relation Sujet-Objet). Pourtant, les comportements de santé possèdent une dimension éminemment sociale, puisque ce sont simultanément des comportements individuels et sociaux (e.g. comportements influencés par les normes et les valeurs d'un groupe social). Pensées dans cette perspective, les compétences psychosociales s'acquièrent et se mobilisent nécessairement dans un champ de communications et de relations interpersonnelles et sociales. En cela, nous rejoignons les approches socio-cognitives qui définissent l'apprentissage comme une construction qui dépend des interactions sociales [77], ainsi que les approches pédagogiques qui s'appuient sur cette notion d'apprentissage social pour conceptualiser l'évaluation et le développement des compétences psychosociales en situation d'ETP [5, 78]. Dans cette perspective, l'approche psychosociale contribuerait à les considérer en relation avec les normes sociales, les insertions sociales et les conditions sociales d'existence dans lesquelles se situent les individus (pour un exemple d'application, voir l'article de Denise Jodelet qui porte sur l'intérêt de la théorie des représentations sociales en situation d'ETP [79]).

L'étude des compétences psychosociales à travers une approche psychosociale présente un intérêt conceptuel, méthodologique et pratique dans le domaine de l'ETP :

- Conceptuel : il s'agit de comprendre la dynamique psychosociale en jeu dans le développement de ces compétences et dans l'adoption de comportements de santé. Cet enjeu conceptuel est important car la transdisciplinarité entre psychologie et santé publique contribue non seulement à éclairer les relations entre les variables psychologiques et les comportements de santé

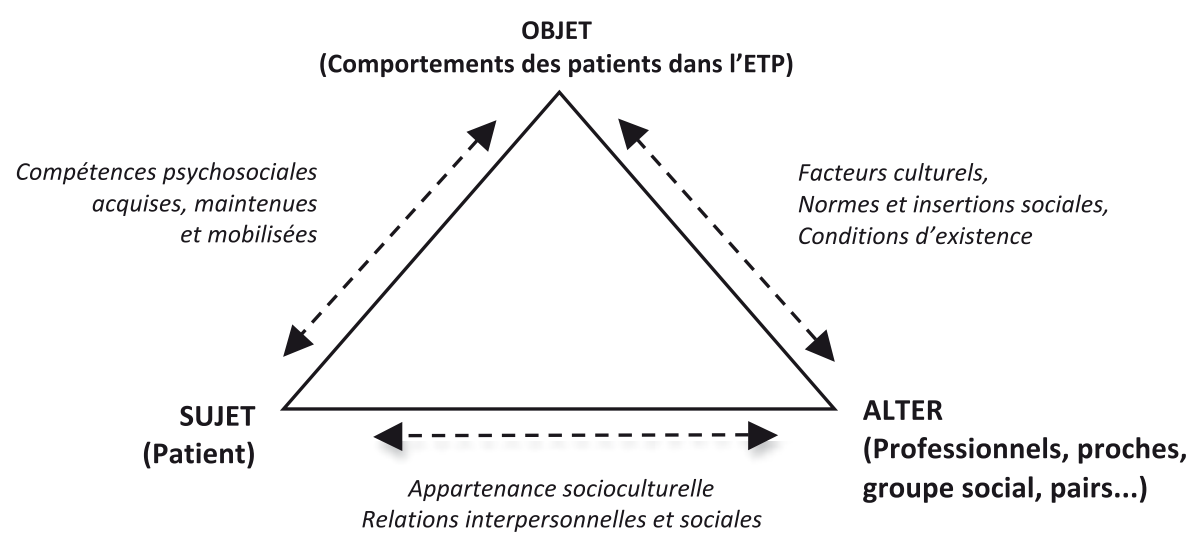

Figure 3 : Le regard psychosocial appliqué à l'éducation du patient 
(e.g. rôle des processus psychosociaux en jeu dans la relation entre le patient et son environnement social sur l'adoption de comportements d'autosoin), mais également à montrer l'importance de prendre en compte des facteurs non sanitaires pour conceptualiser la santé [80].

- Méthodologique : cette approche permettrait de disposer d'un éventail d'indicateurs psychosociaux pertinents pour opérationnaliser les compétences psychosociales et pour mesurer l'efficacité des interventions dans l'acquisition de ces compétences (e.g. identification et sentiment d'appartenance à un groupe social, modalités d'adaptation et de participation sociale, type de contrôle perçu).

- Pratique : cette piste conceptuelle pourrait déboucher sur l'apport de préconisations pour les professionnels qui participent à la conception des interventions d'ETP, à la fois en termes de diagnostic et d'intervention. Par exemple, en élaborant des dispositifs pédagogiques qui visent l'acquisition de certaines compétences psychosociales en fonction de la situation sociale des patients. Ou encore en construisant des outils de mesure ad hoc aux situations de terrain pour optimiser la procédure d'évaluation des dispositifs.

\section{Conclusion}

Les compétences psychosociales en ETP renvoient à un défi conceptuel et pragmatique important. Afin d'être plus efficace dans l'opérationnalisation de ces compétences, il serait nécessaire de réaliser des recherches visant à conceptualiser plus précisément la notion de compétence psychosociale. Enfin, il pourrait être intéressant d'ouvrir ce travail de revue de la littérature à d'autres champs pathologiques et contextes populationnels, comme par exemple de questionner la spécificité des compétences psychosociales à développer en fonction de la population-cible, selon qu'il s'agisse d'enfants, d'adolescents ou d'adultes.

Aucun conflit d'intérêt déclaré

\section{Références}

1. Argyle M. Les compétences sociales. In : Moscovici S, éditeur. Psychologie sociale des relations à autrui. Paris : Nathan; 1994. pp. 87-118.
2. World Health Organization (WHO). Programme on Mental Health. Life Skills Education in School. Genève: WHO; 1994. 54 p.

3. World Health Organization (WHO). Skills for health. Skills-based health education including life skills: an important component of a Child-Friendly/Heal-Promoting School. Genève : WHO ; 2003. 90 p. (Information series on school health; $n^{\circ}$ 9). [Visité le 18/11/2014]. En ligne : http://www.who.int/school_youth_health/media/en/ sch_skills4health_03.pdf.

4. Haute autorité de santé (HAS), Institut national de prévention et d'éducation pour la santé (Inpes). Structuration d'un programme d'éducation thérapeutique du patient dans le champ des maladies chroniques: guide méthodologique. Saint-Denis: HAS; 2007. 112 p. [Visité le 18/11/2014]. En ligne: http://www.has-sante.fr/ portail/upload/docs/application/pdf/etp_-_guide_version_ finale_2_pdf.pdf.

5. D'Ivernois JF, Gagnayre R. Compétences d'adaptation à la maladie du patient : une proposition. Ther Patient Educ. 2011;3(2):201-5.

6. Norris SL, Engelgau MM, Narayan KM. Effectiveness of selfmanagement training in type 2 diabetes: a systematic review of randomized controlled trials. Diabetes Care. 2001;24(3):561-87. doi: 10.2337/diacare.24.3.561.

7. Cooper H, Booth K, Fear S, Gill G. Chronic disease patient education: lessons from meta-analyses. Patient Educ Couns. 2001;44(2):107-17.

8. Warsi A, Wang PS, LaValley MP, Avorn J, Solomon DH. Selfmanagement education programs in chronic disease: a systematic review and methodological critique of the literature. Arch Intern Med. 2004;164(15):1641-9. doi:10.1001/archinte.164.15.1641.

9. Aguilar MJ, Garcia PA, Gonzalez E, Perez MC, Padilla CA. A nursing educational intervention helped by One Touch UltraSmart improves monitoring and glycated haemoglobin levels in type I diabetic children. J Clin Nurs. 2012;21(7-8):1024-32. doi: 10.1111/j. 1365-2702.2011.03926.x.

10. Amsberg S, Anderbro T, Wredling R, Lisspers J, Lins PE, Adamson U, et al. A cognitive behavior therapy-based intervention among poorly controlled adult type 1 diabetes patients--a randomized controlled trial. Patient Educ Couns. 2009;77(1):72-80.

11. Araszkiewicz A, Zozulinska-Ziolkiewicz D, Trepinska M, WieruszWysocka B. Knowledge after five-day teaching program in intensive insulin therapy performed at the onset of type 1 diabetes influence the development of late diabetic complications. Diabetes Res Clin Pract. 2008;81(1):61-7.

12. Attari A, Sartippour $M$, Amini $M$, Haghighi S. Effect of stress management training on glycemic control in patients with type 1 diabetes. Diabetes Res Clin Pract. 2006;73(1):23-8.

13. Benhamou PY, Garnier C, Debaty I, Rueff A, Gilbert C, Ressel M, et al. Basal insulin dose in 40 type 1 diabetic patients remains stable 1 year after educational training in flexible insulin therapy. Diabetes Metab. 2010;36(5):369-74. doi: 10.1016/j.diabet.2010.03.006.

14. Booker S, Morris M, Johnson A. Empowered to change: evidence from a qualitative exploration of a user-informed psycho-educational programme for people with type 1 diabetes. Chronic Illn. 2008;4(1):41-53.

15. Bruttomesso D, Costa S, Dal Pos M, Crazzolara D, Realdi G, Tiengo A, et al. Educating diabetic patients about insulin use: change over time in certainty and correctness of knowledge. Diabetes Metab. 2006;32(3):256-61.

16. Cabrera SM, Srivastava NT, Behzadi JM, Pottorff TM, DiMeglio LA, Walvoord EC. Long-term glycemic control as a result of initial education for children with new onset type 1 diabetes: Does the setting matter? The Diabetes Educator. 2013;39(2):187-94. 
17. Couper JJ, Taylor J, Fotheringham MJ, Sawyer M. Failure to maintain the benefits of home-based intervention in adolescents with poorly controlled type 1 diabetes. Diabetes Care. 1999; 22(12):1933-7.

18. Cox DJ, Gonder-Frederick L, Polonsky W, Schlundt D, Kovatchev B, Clarke W. Blood glucose awareness training (BGAT-2): long-term benefits. Diabetes Care. 2001;24(4):637-42.

19. Cox DJ, Kovatchev B, Koev D, Koeva L, Dachev S, Tcharaktchiev D, et al. Hypoglycemia anticipation, awareness and treatment training (HAATT) reduces occurrence of severe hypoglycemia among adults with type 1 diabetes mellitus. Int J Behav Med. 2004;11(4):212-8.

20. Cox D, Ritterband L, Magee J, Clarke W, Gonder-Frederick L. Blood glucose awareness training delivered over the internet. Diabetes Care. 2008;31(8):1527-8. doi: 10.2337/dc07-1956.

21. Dinneen SF, O'Hara MC, Byrne M, Newell J, Daly L, O'Shea D, et al. The Irish DAFNE study protocol: a cluster randomised trial of group versus individual follow-up after structured education for type 1 diabetes. Trials. 2009;10:88. doi: 10.1186/1745-6215-10-88.

22. Forlani G, Zannoni C, Tarrini G, Melchionda N, Marchesini G. An empowerment-based educational program improves psychological well-being and health-related quality of life in Type 1 diabetes. J Endocrinol Invest. 2006;29(5):405-12.

23. Fritsche A, Stumvoll M, Renn W, Schmulling RM. Diabetes teaching program improves glycemic control and preserves perception of hypoglycemia. Diabetes Res Clin Pract. 1998;40(2):129-35.

24. García-Pérez L, Perestelo-Pérez L, Serrano-Aguilar P, Del Mar TrujilloMartín M. Effectiveness of a psychoeducative intervention in a summer camp for children with type 1 diabetes mellitus. Diabetes Educ. 2010;36(2):310-7.

25. George JT, Valdovinos AP, Russell I, Dromgoole P, Lomax S, Torgerson DJ, et al. Clinical effectiveness of a brief educational intervention in Type 1 diabetes: results from the BITES (Brief Intervention in Type 1 diabetes, Education for Self-efficacy) trial. Diabet Med. 2008;25(12):1447-53. doi: 10.1111/j.1464-5491.2008.02607.x.

26. Grey M, Boland EA, Davidson M, Yu C, Tamborlane WV. Coping skills training for youths with diabetes on intensive therapy. Appl Nurs Res. 1999;12(1):3-12.

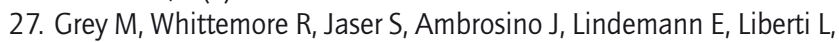
et al. Effects of coping skills training in school-age children with type 1 diabetes. Res Nurs Health. 2009;32(4):405-18. doi: 10.1002/ nur.20336.

28. Hains AA, Davies WH, Parton E, Totka J, Amoroso-Camarata J. A stress management intervention for adolescents with type 1 diabetes. Diabetes Educ. 2000;26(3):417-24.

29. Hermanns N, Kulzer B, Kubiak T, Krichbaum M, Haak T. The effect of an education programme (HyPOS) to treat hypoglycaemia problems in patients with type 1 diabetes. Diabetes Metab Res Rev. 2007; 23(7):528-38. doi: 10.1002/dmrr.710.

30. Hernandez CA, Williamson KM. Evaluation of a self-awareness education session for youth education with type 1 diabetes. Pediatr Nurs. 2004;30(6):459-64,502.

31. Hernandez CA, Hume MR, Rodger NW. Evaluation of a self-awareness intervention for adults with type 1 diabetes and hypoglycemia unawareness. Can J Nurs Res. 2008;40(3):38-56.

32. Hopkins D, Lawrence I, Mansell P, Thompson G, Amiel S, Campbell M, et al. Improved biomedical and psychological outcomes 1 year after structured education in flexible insulin therapy for people with type 1 diabetes: the UK DAFNE experience. Diabetes Care. 2012; 35(8):1638-42. doi: 10.2337/dc11-1579.
33. Howe CJ, Jawad AF, Tuttle AK, Moser JT, Preis C, Buzby M, et al. Education and telephone case management for children with type 1 diabetes: a randomized controlled trial. J Pediatr Nurs. 2005;20(2): 83-95.

34. Howorka K, Pumprla J, Wagner-Nosiska D, Grillmayr H, Schlusche C, Schabmann A. Empowering diabetes out-patients with structured education: short-term and long-term effects of functional insulin treatment on perceived control over diabetes. J Psychosom Res. 2000;48(1):37-44.

35. Jansa M, Vidal M, Viaplana J, Levy I, Conget I, Gomis R, et al. Telecare in a structured therapeutic education programme addressed to patients with type 1 diabetes and poor metabolic control. Diabetes Res Clin Pract. 2006;74(1):26-32.

36. Kinsley BT, Weinger K, Bajaj M, Levy CJ, Simonson DC, Quigley M, et al. Blood glucose awareness training and epinephrine responses to hypoglycemia during intensive treatment in type 1 diabetes. Diabetes Care. 1999;22(7):1022-8.

37. Kubiak T, Hermanns N, Schreckling HJ, Kulzer B, Haak T. Evaluation of a self-management-based patient education program for the treatment and prevention of hypoglycemia-related problems in type 1 diabetes. Patient Educ Couns. 2006;60(2):228-34.

38. Lagger G, Golay A. A 5 dimension therapeutic patient education for type 1 diabetic patients. Ther Patient Educ. 2010;2(2):117-24.

39. Lawson ML, Cohen N, Richardson C, Orrbine E, Pham B. A randomized trial of regular standardized telephone contact by a diabetes nurse educator in adolescents with poor diabetes control. Pediatr Diabetes. 2005;6(1):32-40.

40. Lemozy-Cadroy S, Crognier S, Gourdy P, Chauchard MC, Chale JP, Tauber Dagger JP, et al. Intensified treatment of type 1 diabetes: prospective evaluation at one year of a therapeutic patient education programme. Diabetes Metab. 2002;28(4 Pt 1):287-94.

41. Liesenfeld B, Renner R, Neese M, Hepp KD. Telemedical care reduces hypoglycemias and improves glycemic control in children and adolescents with type 1 diabetes. Diabetes Technol Ther. 2000;2(4): 561-7.

42. Mannucci E, Pala L, Rotella CM. Long-term interactive group education for type 1 diabetic patients. Acta Diabetol. 2005;42(1):1-6.

43. Muller N, Kloos C, Samann A, Wolf G, Muller UA. Evaluation of a treatment and teaching refresher programme for the optimization of intensified insulin therapy in type 1 diabetes. Patient Educ Couns. 2013;93(1):108-13

44. Nansel TR, lannotti RJ, Simons-Morton BG, Cox C, Plotnick LP, Clark LM, et al. Diabetes personal trainer outcomes: short-term and 1-year outcomes of a diabetes personal trainer intervention among youth with type 1 diabetes. Diabetes Care. 2007;30(10):2471-7.

45. Nicholas DB, Fellner KD, Frank M, Small M, Hetherington $R$, Slater $R$, et al. Evaluation of an online education and support intervention for adolescents with diabetes. Soc Work Health Care. 2012;51(9): 815-27.

46. Nordfeldt S, Johansson C, Carlsson E, Hammersjo JA. Persistent effects of a pedagogical device targeted at prevention of severe hypoglycaemia: a randomized, controlled study. Acta Paediatr. 2005;94(10):1395-401.

47. Nunn E, King B, Smart C, Anderson D. A randomized controlled trial of telephone calls to young patients with poorly controlled type 1 diabetes. Pediatr Diabetes. 2006;7(5):254-9.

48. Pélicand J, Gagnayre R, Sandrin-Berthon B, Aujoulat I. A therapeutic education programme for diabetic children: recreational, creative methods, and use of puppets. Patient Educ Couns. 2006;60(2):152-63. 
49. Piana N, Maldonato A, Bloise D, Carboni L, Careddu G, Fraticelli E, et al. The narrative-autobiographical approach in the group education of adolescents with diabetes: a qualitative research on its effects. Patient Educ Couns. 2010;80(1):56-63.

50. Plank J, Kohler G, Rakovac I, Semlitsch BM, Horvath K, Bock G, et al. Long-term evaluation of a structured outpatient education programme for intensified insulin therapy in patients with Type 1 diabetes: a 12-year follow-up. Diabetologia. 2004;47(8):1370-5.

51. Povlsen L, Olsen B, Ladelund S. Educating families from ethnic minorities in type 1 diabetes--experiences from a Danish intervention study. Patient Educ Couns. 2005;59(2):164-70.

52. Povlsen L, Ringsberg KC. Learning to live with type 1 diabetes from the perspective of young nonwestern immigrants in Denmark. J Clin Nurs. 2008;17(11c):300-9.

53. Qayyum AA, Lone SW, Ibrahim MN, Atta I, Raza J. Effects of diabetes self-management education on glycaemic control in children with insulin-dependent diabetes mellitus. J Coll Physicians Surg Pak. 2010;20(12):802-5.

54. Rankin D, Cooke DD, Elliott J, Heller SR, Lawton J. Supporting selfmanagement after attending a structured education programme: a qualitative longitudinal investigation of type 1 diabetes patients' experiences and views. BMC Public Health. 2012;12:652. doi: 10.1186/1471-2458-12-652.

55. Santiprabhob J, Likitmaskul S, Kiattisakthavee P, Weerakulwattana $P$, Chaichanwattanakul K, Nakavachara P, et al. Glycemic control and the psychosocial benefits gained by patients with type 1 diabetes mellitus attending the diabetes camp. Patient Educ Couns. 2008;73(1):60-6.

56. Schachinger $H$, Hegar K, Hermanns N, Straumann M, Keller U, FehmWolfsdorf $G$, et al. Randomized controlled clinical trial of Blood Glucose Awareness Training (BGAT III) in Switzerland and Germany. J Behav Med. 2005;28(6):587-94.

57. Smith BK, Frost J, Albayrak M, Sudhakar R. Facilitating narrative medical discussions of type 1 diabetes with computer visualizations and photography. Patient Educ Couns. 2006;64(1-3):313-21.

58. Snoek FJ, van der Ven NC, Lubach CH, Chatrou M, Ader HJ, Heine RJ, et al. Effects of cognitive behavioural group training (CBGT) in adult patients with poorly controlled insulin-dependent (type 1) diabetes: a pilot study. Patient Educ Couns. 2001;45(2):143-8.

59. Speight J, Amiel SA, Bradley C, Heller S, Oliver L, Roberts S, et al. Long-term biomedical and psychosocial outcomes following DAFNE (Dose Adjustment For Normal Eating) structured education to promote intensive insulin therapy in adults with sub-optimally controlled Type 1 diabetes. Diabetes Res Clin Pract. 2010;89(1):22-9.

60 . Verbeek S, Vos RC, Mul D, Houdijk ME. The influence of an educational program on the $\mathrm{HbA}(1 \mathrm{c})$-level of adolescents with type 1 diabetes mellitus: a retrospective study. J Pediatr Endocrinol Metab. 2011;24(1-2):15-9.

61. Viklund GE, Rudberg S, Wikblad KF. Teenagers with diabetes: selfmanagement education and training on a big schooner. Int J Nurs Pract. 2007;13(6):385-92.

62. Viklund G, Ortqvist E, Wikblad K. Assessment of an empowerment education programme. A randomized study in teenagers with diabetes. Diabet Med. 2007;24(5):550-6.

63. von Sengbusch S, Müller-Godeffroy E, Häger S, Reintjes R, Hiort O, Wagner V. Mobile diabetes education and care: intervention for children and young people with Type 1 diabetes in rural areas of northern Germany. Diabet Med. 2006 23(2):122-7.

64. Waller H, Eiser C, Knowles J, Rogers N, Wharmby S, Heller S, et al. Pilot study of a novel educational programme for 11-16 year olds with type 1 diabetes mellitus: the KICk-OFF course. Arch Dis Child. 2008; 93(11): 927-31. doi: 10.1136/adc.2007.132126.

65. Wdowik MJ, Kendall PA, Harris MA, Keim KS. Development and evaluation of an intervention program: "Control on Campus". Diabetes Educ. 2000;26(1):95-104.

66. Whittemore R, Grey $M$, Lindemann E, Ambrosino J, Jaser S. Development of an Internet coping skills training program for teenagers with type 1 diabetes. Comput Inform Nurs. 2010;28(2):103-11. doi: 10.1097/NCN.0b013e3181cd8199.

67. Whittemore R, Jaser SS, Jeon S, Liberti L, Delamater A, Murphy K, et al. An internet coping skills training program for youth with type 1 diabetes: six-month outcomes. Nurs Res. 2012;61(6):395-404. doi: 10.1097/NNR.0b013e3182690a29.

68. Zoffmann V, Lauritzen T. Guided self-determination improves life skills with type 1 diabetes and $\mathrm{A} 1 \mathrm{C}$ in randomized controlled trial. Patient Educ Couns. 2006;64(1-3):78-86.

69. D'Ivernois JF. Évaluer l'éducation thérapeutique : un défi ? Revue médicale suisse. 2004;2484:1176. [Visité le 18/11/2014]. En ligne : http://titan.medhyg.ch/mh/infos/article.php3?sid=1377.

70. Jodelet $D$. Aperçus sur les méthodologies qualitatives. In: Moscovici S, Buschini F, éditeurs. Les méthodes des sciences humaines. Paris : Presses universitaires de France ; 2003. pp. 139-62. (Fondamental).

71. Cooper H, Booth K, Fear S, Gill G. Chronic disease patient education: lessons from meta-analyses. Patient Educ Couns. 2001;44:107-17.

72. Idier L, Untas A, Aguirrezabal M, Larroumet N, Rascle N, Chauveau P. Quel rôle le psychologue peut-il avoir en éducation thérapeutique en néphrologie? Un exemple en auto-dialyse. Nephrol Ther. 2013;9(3):166-70.

73. Léger P, Garnier PH, Bauer D, Pialoux V, Le Hélias L, Iguenane J, Greffier C. Expériences de psychologues cliniciennes en éducation thérapeutique. Ther Patient Educ. 2012;4:23-8.

74. Contandriopoulos AP, Rey L, Brousselle A, Champagne F. Évaluer une intervention complexe: enjeux conceptuels, méthodologiques et opérationnels. Can J Program Eval. 2012;26(3):1-16.

75. Morin M, Apostolidis T. Contexte social et santé. In : Fischer GN, éditeur. Traité de psychologie de la santé. Paris : Dunod; 2002. pp. 463-89. (Psycho sup).

76. Moscovici S. Psychologie sociale. Paris: Presses universitaires de France; 1998. Le domaine de la psychologie sociale; pp. 5-22.

77. Joannert, P. Compétences et socioconstructivisme: un cadre théorique. Bruxelles: de Boeck Universités; 2002. 100 p. (Perspectives en éducation et formation).

78. Gagnayre R, Marchand C, Pinosa C, Brun MC, Billot D, Iguenane J. Approche conceptuelle d'un dispositif d'évaluation pédagogique du patient. Pédagogie médicale. 2006;7:31-42.

79. Jodelet D. La place des représentations sociales dans l'éducation thérapeutique. Éducation Permanente. 2013;195(2):37-46.

80. McBeth J, Cordingley L. Current issues and new direction in psychology and health: epidemiology and health psychology-please bridge the gap. Psychol Health. 2009;24(8):861-5. 